and University of Bern in Switzerland have now achieved this level of electron bunch charge by fabricating large-area metallic field emitter array cathodes. Their field emitter array comprised $1.5-\mu \mathrm{m}$-base and 5 - $\mu \mathrm{m}$-pitch emitters with a tip apex radius of $10 \mathrm{~nm}$. They arranged a total of $1.2 \times 10^{5}$ emitter tips in a $2.2-\mathrm{mm}$-diameter circle and installed the array in a vacuum chamber. Using a polarized laser beam with a central wavelength of $800 \mathrm{~nm}$ and a pulse duration of $50 \mathrm{fs}$, the researchers illuminated the field emitter array at an incident angle of $60^{\circ}$ normal to the chip surface. They found that the electron emission from the field emitter array rose dramatically when a gate voltage of larger than $70 \mathrm{~V}$ was applied and the incident light was p-polarized and had an intensity of $72 \mathrm{GW} \mathrm{cm}^{-2}$. Under the optimum conditions of a laser intensity of $86 \mathrm{GW} \mathrm{cm}^{-2}$ and a gate voltage of $92 \mathrm{~V}$, the laser-induced charge generated was $5.2 \mathrm{pC}$, which corresponds to $10^{7}$ electrons per laser pulse.

OPTICAL CAVITIES

\section{Vibration-free cube}

Opt. Lett. 36, 3572-3574 (2011)

Optical cavities are essential to the operation of atomic clocks, where they enable highresolution spectroscopy and provide short-term stability during interrogation of the atomic transition. Because the lasing frequency of a cavity is defined by its length, any vibration- or inertia-induced length perturbations must be minimized to achieve spectral purity. Now, Stephen Webster and Patrick Gill at the National Physical Laboratory in the UK have designed a rigidly mounted cubic optical cavity that is insensitive to both inertial and compressive forces. Their design is based on a cubic geometry with four supports placed symmetrically about the optical axis in a tetrahedral configuration. The researchers measured the inertial force sensitivity of their device by locking a laser to the cavity while simultaneously inverting it about three orthogonal axes. They measured a maximum acceleration sensitivity of $2.5 \times 10^{-11} \mathrm{~g}^{-1}$, which is the lowest ever passive sensitivity reported for an optical cavity. The researchers say that the sensitivity may be reduced even further through the use of active feed-forward correction.

NONLINEAR OPTICS

\section{Shaping the deep-UV}

Appl. Phys. B 105, 255-261 (2011)

The ability to temporally shape high-energy UV pulses is important for modern largescale femtosecond X-ray sources such as
X-ray free-electron lasers, where the temporal pulse profile is directly correlated with the longitudinal electron bunch density. Simulations indicate that the longitudinal flat-top intensity profile is one of the most favourable shapes for maintaining the high brilliance of an electron beam during transportation from the electron gun to the linear accelerator. However, the generation of flat-top or arbitrarily shaped high-energy pulses in the $250-300 \mathrm{~nm}$ range remains a challenge. Alexandre Trisorio and co-workers in Switzerland have now combined a highly efficient double-prism stretcher with an acousto-optic programmable dispersive filter applied directly in the UV spectral region to demonstrate the temporal shaping of $270 \mathrm{~nm}$ pulses at energies of up to $37 \mu \mathrm{J}$. The technique enables the generation of highly resolved pulse shapes with flat-top, asymmetric and 'M-shaped' temporal profiles. Although the scheme is limited by two-photon absorption, it nevertheless represents a flexible and efficient method for arbitrarily shaping the phase and amplitude of high-energy picosecond UV pulses. The researchers say that their design could have important applications for free-electron lasers that demand complex temporal pulse shapes in the picosecond regime.

\section{OPTOELECTRONICS}

\section{Graphene photodetection}

Nature Comm. 2, 458 (2011)

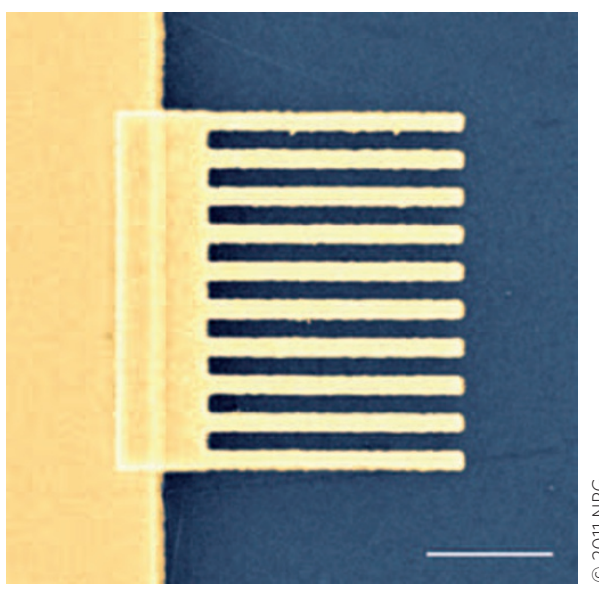

Scientists from the University of Cambridge and the University of Manchester in the UK have shown that introducing goldtitanium plasmonic nanostructures next to the electrical contacts of a graphene photodetector can significantly enhance its maximum voltage. The role of the nanostructures is to covert incident light into plasmonic oscillations and guide electromagnetic energy to the detector's $\mathrm{p}-\mathrm{n}$ junction. The team experimented with several geometries of metal nanostructures, including arrays of double or single dots and a comb (110-nm-wide strips arranged with a pitch of $300 \mathrm{~nm}$ ), which gave the best performance overall. They scanned a focused low-power $(30 \mu \mathrm{W})$ laser beam across the surface of the photodetector and measured the corresponding photovoltage at each point. Illuminating the nanostructures at a laser wavelength matching their plasmon resonance $(514 \mathrm{~nm})$ caused the photovoltage to increase by up to a factor of 20. By employing nanostructures of different shapes and sizes, the researchers were able to tune the detector's wavelength response and polarization sensitivity, thus providing the photodetector with additional flexibility.

\section{RANDOM LASERS \\ Plasmonic feedback \\ Nano Lett. 11, 4295-4298 (2011)}

The localized surface plasmon resonances of metallic nanoparticles have so far been used to enhance lasing in random lasers, but not to provide feedback. Tianrui Zhai and co-workers from Beijing University of Technology in China have now demonstrated a waveguide-plasmonic feedback scheme that offers high-quality confinement of the radiation for efficient amplification, low-threshold lasing and directional output. The scheme is essentially a waveguide on a silica substrate and comprises a $20 \mathrm{~mm} \times 20 \mathrm{~mm}$ layer of randomly distributed gold nano-island structures spin-coated with a solution of poly(methyl methacrylate) (PMMA) doped with a red dye. The researchers chose the dye and nano-islands such that there was an excellent overlap between the photoluminescence spectrum of the dye and the plasmonic resonance spectrum of the gold nano-islands. When pumped by a green $(532 \mathrm{~nm})$ pulsed laser operating with a pulse energy of $50 \mathrm{~mJ}$, a large part of the scattered light was reflected totally at the PMMA-air interface and subsequently scattered by the gold nanostructures within the active waveguide, leading to amplification by stimulated emission in a manner similar to that of a conventional microcavity. The team observed that random lasing commenced at a pump threshold of around $0.1 \mathrm{MW} \mathrm{cm} \mathrm{cm}^{-2}$. Because the lasing wavelength can be tuned by changing the size of the gold nano-islands, the researchers say that multicolour or even white light may be achievable.

Written by James Baxter, Oliver Graydon, Noriaki Horiuchi, David Pile and Rachel Won. 